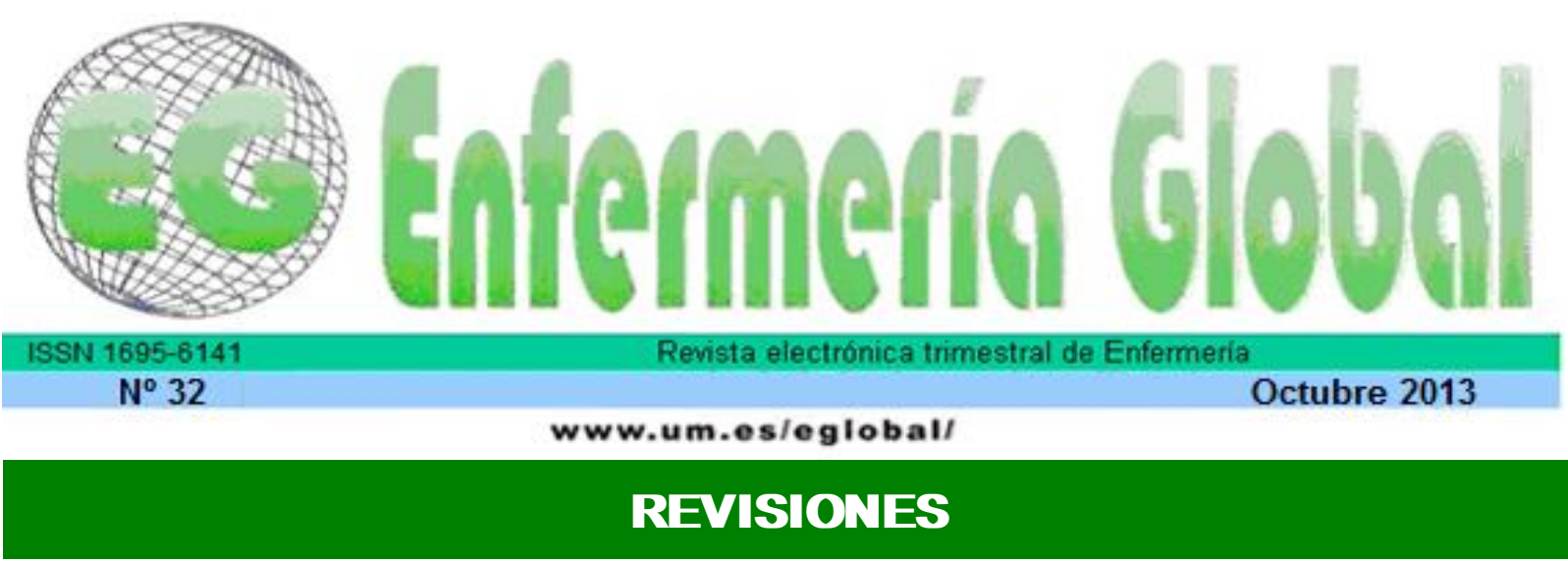

\title{
Estudios sobre adherencia al tratamiento de la malaria
}

Estudos sobre adesão ao tratamento da malária

Studies on adherence to malaria treatment

\section{${ }^{*}$ Almeida Oliveira Reiners, Annelita ${ }^{* *}$ Bianchi Bilo, Bruna ${ }^{* * *}$ de Souza Azevedo, Rosemeiry Capriata ${ }^{* * * *}$ Fernandes Fontes, Cor Jesus

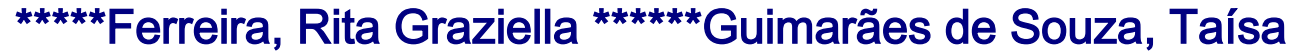

\section{*Doctora en Enfermería. Docente del Departamento en Enfermería y del Programa de Pos-Graduación en Enfermería. E-mail: reiners17@hotmail.com}

(Red Malaria Mato Grosso - Proyecto Malaria por Plasmodium vivax: investigación de factores asociados a la gravedad y a fallos terapéuticos en áreas endémicas de la Amazonia.Órgano financiador: Consejo Nacional de Desarrollo Científico y Tecnológico $-\mathrm{CNPq}$ - proceso $\mathrm{n}^{\circ}$ 555652/2009-2/PRONEX- Red Malaria. Artículo elaborado a partir de la disertación Adherencia de pacientes al tratamiento de la malaria en un municipio endémico de la Amazonia brasileña presentado al Programa de Pos-Graduación en Enfermería, de la Facultad de Enfermería, de la Universidad Federal de Mato Grosso. Cuiabá-MT, Brasil. 2012)

Palabras clave: malaria; terapéutica; adhesión al tratamiento.

Palavras chave: malária; terapêutica; adesão ao tratamento.

Keywords: Malaria; medication adherence; therapy.

\section{RESUMEN}

Objetivo: Estudio de revision que analizó la investigación sobre la adherencia al tratamiento de la malaria producida entre 2000-2011.

Métodos: 27 artículos se obtuvieron a partir de la busca en varias bases de datos, utilizando la combinación de diversos descriptores relacionados con la adherencia al tratamiento y a la malaria.

Resultados: La mayoría de los estudios se realizó en África y América Latina y el tipo más investigado de la malaria fue Plasmodium falciparum. Los esquemas terapéuticos más comúnmente utilizados en la investigación fueron la cloroquina y primaquina, y artemeter-lumefantrina y combinaciones de la sulfadoxina-pirimetamina con otras drogas. Los niños fueron las personas más estudiadas. Se emplearon varios métodos para medir la adherencia. Hubo variabilidad en las tasas de prevalencia de 
adherencia/no adherencia. Pocos estudios han buscado conocer los factores que contribuyen a la adherencia/ no adherencia al tratamiento de medicamentos antipalúdicos.

Conclusión: Existen lagunas a ser completadas con la realización de investigaciones para verificar la prevalencia de la adherencia al tratamiento de la malaria en los países latino-americanos.

\section{RESUMO}

Objetivo: Estudo de revisão que analisou as pesquisas sobre adesão ao tratamento da malária produzidas no período 2000-2011.

Métodos: Obteve-se 27 artigos das buscas em várias bases de dados, utilizando a combinação de diversos descritores relacionados à adesão ao tratamento e à malária.

Resultados: A maioria dos estudos foi realizada na África e na América Latina e o tipo de malária mais pesquisado foi o Plasmodium falciparum. Esquema terapêuticos mais usados nas pesquisas foram cloroquina e primaquina, e artemeter-lumefantrina e combinações da sulfadoxina-pirimetamina com outras drogas. As crianças foram os sujeitos mais estudados. Vários métodos para medir a adesão foram empregados. Houve variabilidade nas taxas de prevalência de adesão/não-adesão. Poucos estudos buscaram conhecer os fatores que contribuem para a adesão/não-adesão ao tratamento de drogas antimaláricas.

Conclusão: Existem lacunas a serem preenchidas com a realização de pesquisas que verifiquem a prevalência de adesão ao tratamento da malária nos países latino-americanos.

\section{ABSTRACT}

Objective: Study review that analyzed some research on adherence to treatment of malaria produced during 2000-2011.

Methods: We obtained 27 articles in a search made in several databases, using a combination of several descriptors related to treatment adherence and malaria.

Results: Most studies were conducted in Africa and Latin America and the type of malaria researched was Plasmodium falciparum. Therapeutic regimens commonly used in research were chloroquine and primaquine, and artemether-lumefantrine and sulfadoxine-pyrimethamine combinations with other drugs. The children were the most studied subject. Several methods were used for measuring compliance. There was variability in prevalence rates of adherence / non adherence. Few studies have sought to determine factors that contribute to adherence / nonadherence to antimalarial drugs.

Conclusion: There are gaps that must be filled with research conducted to verify the prevalence of adherence to malaria treatment in Latin American countries.

\section{INTRODUCCIÓN}

La malaria es una de las enfermedades con mayor inicidencia en el mundo, reconocida por la Organización Mundial de la Salud (WHO) como um problema de salud pública que afecta a cerca de 90 países. ${ }^{(1)}$ La enfermedad se considera importante causa de morbimortalidad en niños y adultos, especialmente en países tropicales y en desarrollo. En 2009, 225 millones de personas contrajeron malaria en el mundo, de los que 781.000 murieron $^{(2)}$

En Brasil, aproximadamente $99,9 \%$ de los casos de malaria se concentran en la región de la Amazônia Legal compuesta por los estados de Acre, Amapá, Amazonas, Pará, Rondônia, Roraima, Mato Grosso, Tocantins y parte de Maranhão ${ }^{(3)}$ y acciones 
orientadas a la prevención, control y tratamiento de la enfermedad son preconizadas por el Ministerio de Salud (MS).

El diagnóstico precoz y el tratamiento adecuado es parte fundamental del programa de control de la malaria y está disponible gratuitamente por el MS en todo el territorio brasileño. Los esquemas terapéuticos actualmente recomendados comprenden períodos cortos que van de 7 a 14 días.

Aunque la eficacia terapéutica de los antimaláricos está comprobada, aún son frecuentes fallos de tratamiento y uno de los motivos es la baja adhesión al tratamiento. ${ }^{(4)}$ Las consecuencias de la no adhesión al tratamiento de la malaria incluyen desarrollo de resistencia a las drogas antimaláricas, permanencia de las personas como fuente de transmisión, control inadecuado de la enfermedad y aumento de los costos en salud. El impacto de estas consecuencias es importante en la vida de las personas, familias y sociedad ${ }^{(5-6-7-8-9-10)}$ y compromete las acciones de los servicios / profesionales de salud y gobierno en el sentido de combatir y controlar la enfermedad.

Investigaciones sobre adhesión a diversos tipos de tratamiento han sido realizadas y publicadas en todo el mundo, sin embargo, aunque muchos autores han realizado estudios sobre terapéutica antimalárica, poco se sabe sobre las investigaciones relacionadas con la adhesión al tratamiento de la malaria.

En este sentido, este estudio tuvo como propósito realizar una revisión crítica de las investigaciones sobre adhesión al tratamiento de la malaria producidas en el periodo de 2000 a 2011.

\section{MÉTODOS}

Se trata de una revisión narrativa realizada en las bases de datos LILACS, MEDLINE, ISI WEB OF KNOWLEDGE, EBSCO y SCOPUS. Se utilizaron los siguientes descriptores y estrategia de busca: (adult[mesh] or adult[tiab] or elderly[mesh] or elderly[tiab] or aging[mesh] or aging[tiab] or old people[mesh] or old people[tiab] or child[mesh] or child[tiab]) AND (malaria[mesh] or malaria[tiab]) AND (medication adherence[mesh] or medication adherence[tiab] or patient dropouts[mesh] or patient dropouts[tiab] or patient compliance[mesh] or patient compliance[tiab] or treatment refusal[mesh] or treatment refusal[tiab] or therapeutics[mesh] or therapeutics[tiab] or health education[mesh] or health education[tiab] or population education[mesh] or population education[tiab]).

Se definieron como criterios de inclusión para la lectura de los artículos: ser artículo publicado en periódicos en el periodo 2000 a 2011 y tener como foco la adhesión al tratamiento de la malaria. $Y$ como criterios de exclusión estudios que trataban solamente de medidas de eficacia terapéutica, de adhesión al tratamiento profiláctico, y de evaluación de intervenciones. Inicialmente se encontraron 2159 textos, por la lectura se excluyeron 1951 títulos. De los 208 restantes, 166 textos fueron excluídos por la lectura de los resúmenes y 16 descartados por la lectura íntegra. Al final quedaron 27 artículos a ser analizados.

Se utilizó para la organización y análisis de las referencias un cuadro en el cual seleccionamos los siguientes items: área de publicación, año de publicación, objetivo del estudio, tipo de investigación, lugar del estudio, población investigada, tipo de 
malaria, esquema terapéutico, medida de la adhesión, definición de adhesión, prevalencia de la adhesión y factores que contribuyen a la adhesión y la no adhesión al tratamiento.

El análisis de los resultados se hizo con base en los estudios sobre adhesión al tratamiento.

\section{RESULTADOS}

La distribución de los 27 artículos de esta revisión muestra que en el período comprendido entre los años 2000-2011, la mayoría de ellos fue publicada en periódicos del área médica en los años 2011 (2), 2010 (7), 2009 (6), 2004 (4). La mayor parte de los estudios de adhesión al tratamiento de la malaria fue realizada en África (16) y la menor en Ásia (5). En América Latina se realizaron seis estudios, siendo tres desarrollados en Brasil, dos en Perú y uno en Ecuador (Figura 1).

Figura 1 - Distribución de los artículos por autor, año de publicación y área de publicación. Cuiabá-MT, 2011.

\begin{tabular}{|c|c|c|c|c|}
\hline \multirow[t]{2}{*}{ Textos } & \multirow{2}{*}{$\begin{array}{c}\text { Año de } \\
\text { publicación }\end{array}$} & \multirow[t]{2}{*}{ Área de publicación } & \multicolumn{2}{|c|}{$\begin{array}{c}\text { Lugar de realización del } \\
\text { estudio }\end{array}$} \\
\hline & & & Continente & País \\
\hline I & 2000 & Medicina & $\begin{array}{l}\text { América } \\
\text { Latina }\end{array}$ & Ecuador \\
\hline II & 2010 & Enfermería & $\begin{array}{l}\text { América } \\
\text { Latina }\end{array}$ & Brasil \\
\hline III & 2010 & Medicina & África & Ruanda \\
\hline IV & 2010 & Medicina & África & Tanzania \\
\hline $\mathrm{V}$ & 2010 & Salud Pública y Medicina & África & Serra Leoa \\
\hline VI & 2010 & Medicina & África & Tanzania \\
\hline VII & 2010 & Medicina & Ásia & Tahilandia \\
\hline VIII & 2009 & Medicina & África & Senegal \\
\hline $\mathrm{IX}$ & 2009 & Medicina & África & Malaui \\
\hline $\mathrm{X}$ & 2009 & Salud Pública y Medicina & África & Tanzania \\
\hline $\mathrm{XI}$ & 2009 & Salud Pública y Medicina & África & Tanzania \\
\hline XII & 2009 & Medicina & África & Senegal \\
\hline XIII & 2009 & $\begin{array}{c}\text { Gastroenterología, Salud } \\
\text { Pública y Ciencia Nutricional }\end{array}$ & Ásia & Tahilandia \\
\hline $\mathrm{XIV}$ & 2008 & Salud Pública y Medicina & África & Senegal \\
\hline $\mathrm{XV}$ & 2004 & Salud Pública y Medicina & África & Zambia \\
\hline $\mathrm{XVI}$ & 2004 & Parasitología y Medicina & África & Sudán \\
\hline XVII & 2010 & Medicina & $\begin{array}{l}\text { América } \\
\text { Latina }\end{array}$ & Perú \\
\hline XVIII & 2007 & Medicina & África & Gambia \\
\hline $\mathrm{XIX}$ & 2004 & Medicina & África & Tanzania \\
\hline $\mathrm{XX}$ & 2004 & Medicina & África & Uganda \\
\hline $\mathrm{XXI}$ & 2002 & Salud Pública y Medicina & Ásia & Sri-Lanka \\
\hline XXII & 2003 & Salud Pública y Medicina & $\begin{array}{l}\text { América } \\
\text { Latina }\end{array}$ & Brasil \\
\hline XXIII & 2001 & Salud Pública & $\begin{array}{l}\text { América } \\
\text { Latina }\end{array}$ & Perú \\
\hline
\end{tabular}




\begin{tabular}{|c|c|c|c|c|}
\hline XXIV & 2006 & Medicina & África & Uganda \\
\hline XXV & 2011 & Medicina & Ásia & Malaui \\
\hline XXVI & 2008 & Salud Pública y Medicina & Ásia & Bangladesh \\
\hline XXVII & 2011 & Medicina & $\begin{array}{c}\text { América } \\
\text { Latina }\end{array}$ & Brasil \\
\hline
\end{tabular}

En los estudios revisados, el tipo de malaria más investigado fue el Plasmodium falciparum. En cuanto a los medicamentos, estos fueron variados y combinados de forma y dosis diversas. Algunos estudios utilizaron esquemas de cloroquina + primaquina para infecciones por $P$. vivax. En los casos de malaria por $P$. falciparum, se empleó artemeter + lumefantrina y combinaciones de sulfadoxina + pirimetamina con otras drogas.

La Figura 2 muestra que el principal objetivo de los estudios encontrados fue verificar la adhesión al tratamiento. En algunos estudios los autores tuvieron el propósito de investigar los factores que contribuyen a la adhesión/no adhesión, siendo que en 3 de ellos, este era el único objetivo. Los niños fueron los sujetos más investigados (25 estudios) y sus cuidadores aparecen también como población de interés en 9 de ellos.

Figura 2 - Distribución de los artículos por tipo de investigación, objetivos, población investigada, medida y definición de la adhesión. Cuiabá-MT, 2011.

\begin{tabular}{|c|c|c|c|c|}
\hline Tx & Objetivos & Sujetos & $\begin{array}{c}\text { Medida de la } \\
\text { adhesión }\end{array}$ & $\begin{array}{c}\text { Definición de la } \\
\text { adhesión/no } \\
\text { adhesión }\end{array}$ \\
\hline I & $\begin{array}{c}\text { Verificar la no } \\
\text { adhesión, su } \\
\text { relación con } \\
\text { diversos factores y } \\
\text { trazar el perfil de la } \\
\text { persona que no } \\
\text { adhiere }\end{array}$ & $\begin{array}{c}\text { Pacientes de } \\
\text { todas las } \\
\text { edades y sexo }\end{array}$ & $\begin{array}{c}\text { Auto-relato y } \\
\text { medición de la } \\
\text { concentración en } \\
\text { sangre }\end{array}$ & $\begin{array}{c}\text { Relacionada con el } \\
\text { seguimiento de la } \\
\text { prescripción médica } \\
\text { recomendada }\end{array}$ \\
\hline II & $\begin{array}{c}\text { Descripción del } \\
\text { comportamiento de } \\
\text { los usuarios en } \\
\text { relación al } \\
\text { tratamiento }\end{array}$ & $\begin{array}{c}\text { Pacientes } \\
\text { mayores de } 18 \\
\text { años }\end{array}$ & Auto-relato & $\begin{array}{c}\text { Relacionada con la } \\
\text { declaración del } \\
\text { paciente de } \\
\text { seguimiento del } \\
\text { tratamiento } \\
\text { recomendado }\end{array}$ \\
\hline III & $\begin{array}{c}\text { Testar y comparar } \\
\text { métodos de medida } \\
\text { de la adhesión }\end{array}$ & $\begin{array}{c}\text { Cuidadores de } \\
\text { niños menores } \\
\text { de } 5 \text { años }\end{array}$ & $\begin{array}{c}\text { Auto-relato, } \\
\text { recuento de } \\
\text { medicamentos y } \\
\text { monitorización } \\
\text { electrónica }\end{array}$ & $\begin{array}{c}\text { Relacionada con el } \% \\
\text { de medicamentos } \\
\text { ingeridos }\end{array}$ \\
\hline IV & $\begin{array}{c}\text { Entender el dilema } \\
\text { de los cuidadores } \\
\text { en adherir o no al } \\
\text { tratamiento }\end{array}$ & $\begin{array}{c}\text { Cuidadores de } \\
\text { niños menores } \\
\text { de } 5 \text { años }\end{array}$ & No definió & No definió \\
\hline
\end{tabular}




\begin{tabular}{|c|c|c|c|c|}
\hline V & $\begin{array}{l}\text { Verificar la } \\
\text { adhesión e } \\
\text { identificar las } \\
\text { razones de no } \\
\text { adhesión }\end{array}$ & $\begin{array}{l}\text { Pacientes } \\
\text { mayores de } 1 \\
\text { año de edad }\end{array}$ & $\begin{array}{l}\text { Auto-relato y } \\
\text { recuento de } \\
\text { medicamentos }\end{array}$ & $\begin{array}{l}\text { Relacionada con la } \\
\text { declaración del } \\
\text { paciente de } \\
\text { seguimiento completo } \\
\text { del tratamiento y } \\
\text { confirmada por el } \\
\text { recuento de } \\
\text { medicamentos }\end{array}$ \\
\hline VI & $\begin{array}{l}\text { Evaluar la adhesión } \\
\text { y la aceptabilidad al } \\
\text { tratamiento oficial }\end{array}$ & $\begin{array}{c}\text { Niños mayores } \\
\text { de } 3 \text { años y } \\
\text { menores de } 5 \\
\text { años }\end{array}$ & $\begin{array}{l}\text { Auto-relato y } \\
\text { recuento de } \\
\text { medicamentos }\end{array}$ & No definió \\
\hline VII & $\begin{array}{l}\text { Verificar la } \\
\text { adhesión al } \\
\text { tratamiento }\end{array}$ & $\begin{array}{l}\text { Pacientes de } 4 \\
\text { a } 69 \text { años de } \\
\text { edad }\end{array}$ & $\begin{array}{l}\text { Auto-relato y } \\
\text { medición de la } \\
\text { concentración de } \\
\text { la droga en } \\
\text { sangre }\end{array}$ & $\begin{array}{l}\text { Relacionada con el } \\
\text { seguimiento de la } \\
\text { prescripción médica } \\
\text { recomendada y } \\
\text { límites aceptables de } \\
\text { la droga en sangre }\end{array}$ \\
\hline VIII & $\begin{array}{l}\text { Testar y comparar } \\
\text { métodos de medida } \\
\text { de la adhesión }\end{array}$ & $\begin{array}{c}\text { Niños entre } 2 \text { y } \\
10 \text { años }\end{array}$ & $\begin{array}{c}\text { Auto-relato, } \\
\text { medición de la } \\
\text { concentración de } \\
\text { la droga en orina } \\
\text { y en sangre }\end{array}$ & $\begin{array}{c}\text { Relacionada con el \% } \\
\text { de medicamentos } \\
\text { ingeridos }\end{array}$ \\
\hline IX & $\begin{array}{l}\text { Investigar el } \\
\text { impacto de la baja } \\
\text { adhesión y la } \\
\text { eficacia de tres } \\
\text { tratamientos }\end{array}$ & $\begin{array}{l}\text { Niños mayores } \\
\text { de } 12 \text { meses y } \\
\text { adultos }\end{array}$ & $\begin{array}{l}\text { Auto-relato y } \\
\text { monitorización } \\
\text { electrónica }\end{array}$ & No definió \\
\hline$X$ & $\begin{array}{l}\text { Estimar la adhesión } \\
\text { de cuidadores al } \\
\text { tratamiento oficial e } \\
\text { identificar los } \\
\text { factores asociados } \\
\text { a la adhesión }\end{array}$ & $\begin{array}{c}\text { Cuidadores de } \\
\text { niños menores } \\
\text { de } 5 \text { años }\end{array}$ & $\begin{array}{l}\text { Auto-relato y } \\
\text { recuento de } \\
\text { medicamentos }\end{array}$ & $\begin{array}{l}\text { Relacionada con la } \\
\text { declaración del } \\
\text { paciente de } \\
\text { seguimiento completo } \\
\text { del tratamiento y } \\
\text { confirmada por la } \\
\text { recuento de } \\
\text { medicamentos } \\
\end{array}$ \\
\hline$X I$ & $\begin{array}{c}\text { Describir factores } \\
\text { que influían en los } \\
\text { cuidadores en las } \\
\text { decisiones de } \\
\text { adherir al } \\
\text { tratamiento }\end{array}$ & $\begin{array}{l}\text { Cuidadores de } \\
\text { niños }\end{array}$ & No definió & $\begin{array}{l}\text { Relacionada con el } \\
\text { envío del paciente } \\
\text { para atención } \\
\text { ambulatoria tras el } \\
\text { tratamiento } \\
\text { medicamentoso de } \\
\text { urgencia }\end{array}$ \\
\hline XII & $\begin{array}{l}\text { Medir la adhesión e } \\
\text { identificar factores } \\
\text { relacionados con la } \\
\text { adhesión al } \\
\text { tratamiento }\end{array}$ & $\begin{array}{c}\text { Niños entre } 2 \text { y } \\
10 \text { años }\end{array}$ & Auto-relato & $\begin{array}{c}\text { Relacionada con el \% } \\
\text { de medicamentos } \\
\text { ingeridos }\end{array}$ \\
\hline XIII & $\begin{array}{l}\text { Definir factores que } \\
\text { influyeron en la } \\
\text { adhesión/no } \\
\text { adhesión al } \\
\text { tratamiento }\end{array}$ & $\begin{array}{l}\text { Pacientes } \\
\text { entre } 11 \text { y } 88 \\
\text { años }\end{array}$ & Auto-relato & $\begin{array}{l}\text { Relacionada con el } \\
\text { seguimiento de la } \\
\text { prescripción médica } \\
\text { recomendada }\end{array}$ \\
\hline
\end{tabular}




\begin{tabular}{|c|c|c|c|c|}
\hline XIV & $\begin{array}{l}\text { Examinar las tasas } \\
\text { de adhesión al } \\
\text { tratamiento y su } \\
\text { eficacia en niños }\end{array}$ & $\begin{array}{c}\text { Niños con } \\
\text { edad entre } 2 \text { y } \\
10 \text { años }\end{array}$ & $\begin{array}{c}\text { Auto-relato, } \\
\text { medición de la } \\
\text { concentración de } \\
\text { la droga en orina } \\
\text { y en sangre } \\
\end{array}$ & $\begin{array}{c}\text { Relacionada con el \% } \\
\text { de medicamentos } \\
\text { ingeridos }\end{array}$ \\
\hline$X V$ & $\begin{array}{l}\text { Medir la adhesión } \\
\text { en condiciones } \\
\text { rutinarias de } \\
\text { prescripción y } \\
\text { acceder razones } \\
\text { para la no } \\
\text { adhesión }\end{array}$ & $\begin{array}{l}\text { Niños menores } \\
\text { de } 5 \text { años }\end{array}$ & $\begin{array}{l}\text { Auto-relato y } \\
\text { contagem de } \\
\text { medicamentos }\end{array}$ & $\begin{array}{l}\text { Relacionada con la } \\
\text { declaración del } \\
\text { paciente de } \\
\text { seguimiento completo } \\
\text { del tratamiento y } \\
\text { confirmada por el } \\
\text { recuento de } \\
\text { medicamentos } \\
\end{array}$ \\
\hline $\mathrm{XVI}$ & $\begin{array}{l}\text { Verificar la } \\
\text { adhesión al } \\
\text { tratamiento }\end{array}$ & $\begin{array}{c}\text { Cuidadores de } \\
\text { niños menores } \\
\text { de } 5 \text { años }\end{array}$ & $\begin{array}{l}\text { Auto-relato y } \\
\text { recuento de } \\
\text { medicamentos }\end{array}$ & $\begin{array}{l}\text { Relacionada con la } \\
\text { declaración del } \\
\text { paciente de } \\
\text { seguimiento completo } \\
\text { del tratamiento y } \\
\text { confirmada por el } \\
\text { recuento de } \\
\text { medicamentos } \\
\end{array}$ \\
\hline XVII & $\begin{array}{c}\text { Medir la adhesión y } \\
\text { considerar los } \\
\text { factores que } \\
\text { influyen en la } \\
\text { adhesión }\end{array}$ & $\begin{array}{l}\text { Habitantes de } \\
\text { la Amazonia } \\
\text { Peruana }\end{array}$ & $\begin{array}{c}\text { Auto-relato y } \\
\text { datos obtenidos } \\
\text { del registro del } \\
\text { paciente }\end{array}$ & $\begin{array}{l}\text { Relacionada con el } \\
\text { seguimiento de la } \\
\text { prescripción médica } \\
\text { recomendada }\end{array}$ \\
\hline XVIII & $\begin{array}{l}\text { Verificar el papel } \\
\text { de la comunicación } \\
\text { profesional de } \\
\text { salud/cliente en la } \\
\text { adhesión al } \\
\text { tratamiento } \\
\end{array}$ & $\begin{array}{l}\text { Cuidadores de } \\
\text { niños menores } \\
\text { de } 10 \text { años }\end{array}$ & Auto-relato & No definió \\
\hline XIX & $\begin{array}{l}\text { Medir la adhesión } \\
\text { al tratamiento } \\
\text { oficial y evaluar los } \\
\text { factores que } \\
\text { pueden influir en la } \\
\text { adhesión }\end{array}$ & $\begin{array}{l}\text { Pacientes } \\
\text { mayores e } \\
\text { iguales a } 2 \\
\text { meses }\end{array}$ & $\begin{array}{l}\text { Auto-relato y } \\
\text { recuento de } \\
\text { medicamentos }\end{array}$ & $\begin{array}{l}\text { Relacionada con la } \\
\text { declaración del } \\
\text { paciente de } \\
\text { seguimiento completo } \\
\text { del tratamiento y } \\
\text { confirmada por el } \\
\text { recuento de } \\
\text { medicamentos } \\
\end{array}$ \\
\hline$X X$ & $\begin{array}{l}\text { Medir la adhesión } \\
\text { e identificar } \\
\text { factores de riesgo } \\
\text { para baja adhesión } \\
\text { al tratamiento }\end{array}$ & $\begin{array}{l}\text { Niños menores } \\
\text { de } 5 \text { años, de } \\
5-14 \text { y mayores } \\
\text { o iguales a } 15 \\
\text { años edad. }\end{array}$ & $\begin{array}{l}\text { Auto-relato, } \\
\text { medición de la } \\
\text { concentración de } \\
\text { la droga en } \\
\text { sangre y } \\
\text { recuento de } \\
\text { medicamentos }\end{array}$ & $\begin{array}{l}\text { Relacionada con la } \\
\text { declaración del } \\
\text { paciente de } \\
\text { seguimiento completo } \\
\text { del tratamiento y } \\
\text { confirmada por el } \\
\text { recuento de } \\
\text { medicamentos }\end{array}$ \\
\hline$X X I$ & $\begin{array}{l}\text { Investigar } \\
\text { comportamiento de } \\
\text { busca yadhesión al } \\
\text { tratamiento }\end{array}$ & Adultos y niños & Auto-relato & $\begin{array}{l}\text { Relacionado con la } \\
\text { declaración del } \\
\text { paciente de no } \\
\text { seguimiento completo } \\
\text { del tratamiento }\end{array}$ \\
\hline
\end{tabular}




\begin{tabular}{|c|c|c|c|c|}
\hline XXII & $\begin{array}{c}\text { Describir la } \\
\text { asociación entre el } \\
\text { auto-relato de } \\
\text { adhesión al } \\
\text { tratamiento y la } \\
\text { ocurrencia de } \\
\text { malaria }\end{array}$ & $\begin{array}{l}\text { Pacientes de } \\
\text { varias edades. }\end{array}$ & Auto-relato & No definió \\
\hline XXIII & $\begin{array}{c}\text { Determinar los } \\
\text { factores asociados } \\
\text { a la adhesión al } \\
\text { tratamiento }\end{array}$ & $\begin{array}{l}\text { Profesionales } \\
\text { de salud y } \\
\text { pacientes }\end{array}$ & No definió & No definió \\
\hline XXIV & $\begin{array}{l}\text { Estudar la } \\
\text { adhesión al } \\
\text { tratamiento }\end{array}$ & $\begin{array}{l}\text { Cuidadores de } \\
\text { niños } \leq 5 \text { años }\end{array}$ & $\begin{array}{l}\text { Auto-relato y } \\
\text { recuento de } \\
\text { medicamentos }\end{array}$ & $\begin{array}{l}\text { Relacionada con la } \\
\text { declaración del } \\
\text { paciente de } \\
\text { seguimiento completo } \\
\text { del tratamiento y } \\
\text { confirmada por el } \\
\text { recuento de } \\
\text { medicamentos }\end{array}$ \\
\hline XXV & $\begin{array}{l}\text { Evaluar la adhesión } \\
\text { al tratamiento e } \\
\text { identificar factores } \\
\text { asociados a la } \\
\text { adhesión }\end{array}$ & Adultos y niños & $\begin{array}{l}\text { Auto-relato y } \\
\text { recuento de } \\
\text { medicamentos }\end{array}$ & No definió \\
\hline XXVI & $\begin{array}{l}\text { Determinar la } \\
\text { eficacia del } \\
\text { Artemeter- } \\
\text { Lumefantrine y } \\
\text { medir la adhesión } \\
\text { al tratamiento }\end{array}$ & Adultos y niños & $\begin{array}{l}\text { Auto-relato y } \\
\text { recuento de } \\
\text { medicamentos }\end{array}$ & $\begin{array}{l}\text { Relacionada con la } \\
\text { declaración del } \\
\text { paciente de } \\
\text { seguimiento completo } \\
\text { del tratamiento y } \\
\text { confirmada por el } \\
\text { recuento de } \\
\text { medicamentos }\end{array}$ \\
\hline XXVII & $\begin{array}{c}\text { Estimar la } \\
\text { prevalencia de la } \\
\text { baja adhesión por } \\
\text { P. vivax con } \\
\text { Cloroquina y } \\
\text { Primaquina e } \\
\text { identificar factores } \\
\text { de baja adhesión }\end{array}$ & Adultos y niños & $\begin{array}{l}\text { Auto-relato y } \\
\text { recuento de } \\
\text { medicamentos }\end{array}$ & $\begin{array}{l}\text { Relacionada con la } \\
\text { declaración del } \\
\text { paciente de } \\
\text { seguimiento completo } \\
\text { del tratamiento y } \\
\text { confirmada por el } \\
\text { recuento de } \\
\text { medicamentos }\end{array}$ \\
\hline
\end{tabular}

A fin de medir la adhesión/no adhesión de los pacientes al tratamiento, los autores utilizaron métodos diversos: auto-relato, recuento de medicamentos, monitorización electrónica, medición de la concentración de las drogas en sangre y en orina, y datos obtenidos de los registros de los pacientes. En la Figura 2, es posible percibir que la mayoría de los autores usaron más de un procedimiento para medir la adhesión de los pacientes al tratamiento de la malaria, estando el auto-relato presente en todos los estudios, excepto en los que no tenían como objetivo verificar la adhesión.

La definición operacional más adoptada por los investigadores de los estudios revisados tenía en consideración cómo era medida la adhesión, o sea, si el investigador pretendía medirla por medio del auto-relato se considera que la misma 
adhirió si declaraba haber seguido el tratamiento completo. En algunas investigaciones, la definición se relacionaba también con el seguimiento completo del tratamiento confirmado por el recuento de medicamentos. En 5 estudios, los autores no presentaron la definición de adhesión que adoptaron porque no era su objetivo verificar la adhesión. En 2 estudios, aunque el propósito de los autores fuese verificar la adhesión, no la definieron explícitamente (Figura 2).

En relación a la prevalencia de la adhesión al tratamiento de la malaria, evidenciamos que hubo bastante variabilidad en los resultados. Considerando los diferentes métodos, esquemas terapéuticos y tipos de población estudiada, la prevalencia de adhesión en los niños varió de 37,7 a $96,3 \%$. En adultos y niños, la variabilidad fue de 48,3 a $97,4 \%$.

La Figura 3 muestra que, entre los factores contribuyentes a la adhesión al tratamiento que están relacionados con el paciente, destaca el mayor conocimiento de la enfermedad y la gravedad de los síntomas. Entre los relacionados con los servicios de salud, la dosis supervisionada y la dispensación exacta del número de pastillas. El uso de figuras pictóricas en el embalaje de los antimaláricos fue el factor relacionado con los medicamentos que más contribuyó a la adhesión. Por último, las buenas orientaciones de los profesionales de salud en relación a los medicamentos y sus efectos fue el factor apuntado en los resultados como colaborador en la adhesión al tratamiento.

Igualmente, en la Figura 3 se observa que en los estudios analizados, entre los factores contribuyentes a la no adhesión al tratamiento, en relación al paciente, el olvido y la mejora de los síntomas fueron los que más aparecieron en los resultados. En cuanto a los factores relacionados con los servicios de salud, la dificultad de acceso fue el más apuntado en las investigaciones. En relación a los medicamentos, los efectos colaterales de los antimaláricos fueron los mayores contribuyentes de la no adhesión al tratamiento. Por fin, a falta de orientaciones $\mathrm{y} / \mathrm{u}$ orientaciones incorrectas sobre los fármacos realizadas por el profesional de salud se destacó como factor colaborador de la no adhesión de los pacientes a la terapéutica antimalárica.

Figura 3. Factores contribuyentes a la adhesión y a la no adhesión a los tratamientos encontrados en los estudios analizados.

\begin{tabular}{|c|c|c|c|}
\hline \multicolumn{2}{|c|}{$\begin{array}{c}\text { Factores contribuyentes a la } \\
\text { adhesión al tratamiento }\end{array}$} & \multicolumn{2}{|c|}{$\begin{array}{l}\text { Factores contribuyentes a la no } \\
\text { adhesión al tratamiento }\end{array}$} \\
\hline $\begin{array}{l}\text { Relacionados con el } \\
\text { paciente }\end{array}$ & Textos & Relacionados con el paciente & Textos \\
\hline $\begin{array}{l}\text { Mayor conocimiento de la } \\
\text { enfermedad }\end{array}$ & $\begin{array}{l}1,5,11,13,19,2 \\
3,26\end{array}$ & Olvido & $\begin{array}{l}1,2,5,6,15,1 \\
6,21,24,25, \\
27\end{array}$ \\
\hline $\begin{array}{l}\text { Gravedad de las señales y } \\
\text { síntomas }\end{array}$ & $2,4,11,13$ & Mejora de los síntomas & $\begin{array}{l}1,5,10,12,1 \\
4,15,17,19, \\
21,24,25,26\end{array}$ \\
\hline & & $\begin{array}{l}\text { Falta de comprensión de las } \\
\text { instrucciones }\end{array}$ & $\begin{array}{l}4,6,10,15,1 \\
6,25\end{array}$ \\
\hline $\begin{array}{l}\text { Experiencia previa con la } \\
\text { enfermedad }\end{array}$ & $5,12,26$ & $\begin{array}{l}\text { Guarda o pérdida de los } \\
\text { comprimidos }\end{array}$ & $\begin{array}{l}6,9,15,19,2 \\
5,26\end{array}$ \\
\hline
\end{tabular}




\begin{tabular}{|c|c|c|c|}
\hline $\begin{array}{l}\text { Faja etária (niños) al } \\
\text { cuidado de los padres }\end{array}$ & 10 & $\begin{array}{l}\text { Ingestión o administración errada } \\
\text { de los medicamentos }\end{array}$ & $\begin{array}{l}10,12,14,19 \\
, 21,25\end{array}$ \\
\hline Mayor grado de escolaridad & 10 & Escasez de alimento & $5,16,21$ \\
\hline Confianza en el tratamiento & $2,13,23$ & $\begin{array}{l}\text { Dificultad de percepción en } \\
\text { cuanto a la eficacia de las drogas } \\
\text { y de los servicios de salud }\end{array}$ & $10,21,27$ \\
\hline $\begin{array}{l}\text { Mejora rápida de las } \\
\text { señales y síntomas }\end{array}$ & 20 & $\begin{array}{l}\text { Falta de conocimiento sobre la } \\
\text { malaria }\end{array}$ & 13 \\
\hline Miedo a empeorar & 2 & Baja escolaridad & 1,20 \\
\hline \multirow[t]{5}{*}{ Deseo de mejorar } & 2 & Uso de tratamientos alternativos & 1 \\
\hline & & Baja condición socioeconómica & 4 \\
\hline & & Migração de los pacientes & 23 \\
\hline & & Uso de bebidas alcohólicas & 27 \\
\hline & & $\begin{array}{l}\text { Resistencia del paciente a tomar } \\
\text { la medicación }\end{array}$ & 1,26 \\
\hline $\begin{array}{l}\text { Relacionados con los } \\
\text { servicios de salud }\end{array}$ & Textos & $\begin{array}{c}\text { Relacionados con los servicios } \\
\text { de salud }\end{array}$ & Textos \\
\hline Dosis supervisada & $10,23,25$ & $\begin{array}{l}\text { Dificultad de acceso al servicio } \\
\text { de salud }\end{array}$ & 4,23 \\
\hline Gratuidad del medicamento & 23 & Más calidad de la atención & 11 \\
\hline $\begin{array}{l}\text { Mejor acceso al servicio de } \\
\text { salud }\end{array}$ & 25 & \multirow{4}{*}{$\begin{array}{c}\text { Relacionados con los } \\
\text { medicamentos }\end{array}$} & \\
\hline $\begin{array}{l}\text { Dispensación exacta del } \\
\text { número de pastillas }\end{array}$ & 10,13 & & \\
\hline $\begin{array}{l}\text { Participación de los } \\
\text { profesionales de salud en el } \\
\text { tratamiento }\end{array}$ & 23 & & \\
\hline $\begin{array}{c}\text { Relacionados con los } \\
\text { medicamentos }\end{array}$ & Textos & & Textos \\
\hline $\begin{array}{l}\text { Figuras pictóricas en el } \\
\text { embalaje del medicamento }\end{array}$ & 6,23 & Efectos colaterales & $\begin{array}{l}1,5,6,10,13, \\
14,17,21,23 \\
, 24,25,27\end{array}$ \\
\hline $\begin{array}{l}\text { Medicación de fácil } \\
\text { ingestión }\end{array}$ & 23 & Gusto del medicamento & $1,15,21,24$ \\
\hline $\begin{array}{l}\text { Esquemas terapéuticos } \\
\text { cortos }\end{array}$ & 23 & Medicación de difícil ingestión & 14 \\
\hline $\begin{array}{l}\text { Medicamento en forma de } \\
\text { tableta }\end{array}$ & 14 & \multirow[b]{2}{*}{$\begin{array}{l}\text { Relacionados con el } \\
\text { profesional de salud }\end{array}$} & \\
\hline $\begin{array}{l}\text { Relacionados con el } \\
\text { profesional de salud }\end{array}$ & Textos & & Textos \\
\hline $\begin{array}{l}\text { Buenas orientaciones en } \\
\text { relación a los medicamentos } \\
\text { y sus efectos }\end{array}$ & $\begin{array}{l}5,12,19,25,26 \\
27\end{array}$ & $\begin{array}{l}\text { Falta de orientaciones y/u } \\
\text { orientaciones incorrectas en } \\
\text { relación al medicamento y sus } \\
\text { efectos }\end{array}$ & $5,10,14,15$ \\
\hline
\end{tabular}

\section{DISCUSIÓN}

En este estudio, la amplia revisión de la literatura desarrollada en bases de datos referidas al área de la salud permitió que los resultados presentados aportasen más conocimiento sobre la temática estudiada. Aunque su propósito es solamente 
describir las investigaciones sobre adhesión al tratamiento de la malaria producidas en el período de 2000 a 2011, su importancia está en el hecho de que, hasta el momento, no se han encontrado investigaciones realizadas con este fin.

El creciente número de publicaciones sobre adhesión al tratamiento de la malaria, en los últimos once años, refleja la preocupación que los estudiosos tienen con este problema, principalmente los profesionales del área médica que lidian directamente con la prescripción del tratamiento.

El hecho de que la mayor parte de los estudios se haya desarrollado en el continente africano se justifica por ser en esta región donde se concentran más casos de malaria en el mundo. Se estima que $91 \%$ de los casos, en 2010 , se dio en África, seguido del Sudeste Asiático (6\%) y regiones del Mediterráneo Oriental (3\%). ${ }^{(11)}$

Por otro lado, a pesar de que la malaria afecta a un gran número de personas en América Latina, esta revisión mostró que existen investigaciones publicadas sobre el tema adhesión al tratamiento de la enfermedad siendo imprescindible el desarrollo de investigaciones pertinentes al conocimiento sobre la adhesión/no adhesión al tratamiento de la malaria en los países latino-americanos, pues informaciones sobre el fenómeno permiten saber en qué medida hay éxito en el control de la enfermedad desarrollando acciones para combatir el problema de modo adecuado y eficaz.

El mayor número de estudios sobre la malaria falciparum se justifica por el hecho de que la mayoría fue realizada en África y Ásia, donde la ocurrencia de la enfermedad se da principalmente por ese tipo.

La existencia de muchos estudios que involucran a niños y a sus cuidadores se explica por tratarse de una población de riesgo, con baja inmunidad. De acuerdo con la $\mathrm{WHO}, 86 \%$ de los óbitos por malaria en el mundo fueron niños menores de cinco años, principalmente en África. ${ }^{(11)}$

El propósito de la medida de la adhesión no es meramente certificar si existe o no adhesión del paciente al tratamiento, sino también verificar la efectividad del régimen terapéutico en el combate y control de la enfermedad y la ocurrencia o no de eventos adversos con el advenimiento de la no adhesión.

No existe un criterio único para medir la adhesión al tratamiento. La literatura refiere diversos métodos de medida de la adhesión: auto-relato, concentración de las drogas en orina y en sangre, recuento de los medicamentos, monitorización electrónica de la droga (MEMS) o asociación de los mismos. Cada uno tiene su peculiaridad, ventajas y desventajas. La elección del método a utilizar depende esencialmente del objetivo de la investigación y de los recursos disponibles. ${ }^{(12)}$ El uso de métodos combinados de medida de la adhesión en las investigaciones garantiza mayor confiabilidad a los resultados, una vez que las desvantajas de unos son suplidas por las ventajas del otro.

El hecho de que el auto-relato haya sido el más adoptado por los investigadores se explica por ser un método simple, fácil y de bajo costo, muy utilizado en la práctica clínica. Por ello, puede resultar en una distorsión de la realidad, pues la entrevista puede constreñir al paciente, tornando la información inconsistente. Además, el paciente tiende a decir al profesional lo que él quiere oir, generalmente sobreestimando el seguimiento del régimen terapéutico. Sin embargo, en el caso de 
las investigaciones de adhesión al tratamiento, puede producir buenos indicadores si las preguntas son conducidas de modo no coercitivo y garantizando total sigilo y respeto a los pacientes. ${ }^{(13)}$

Además del auto-relato, el recuento de los medicamentos fue otro método indirecto utilizado en los estudios. Este método tiene la ventaja de ser objetivo, de fácil aplicación y de permitir la comparación del estado clínico del paciente con la cantidad de medicamento retirada del blister/envelope. Sin embargoo, esto no garantiza que el medicamento fue realmente tomado. ${ }^{(7,14)}$ Esta también es la desventaja del método que usa la monitorización electrónica de la droga (MEMS), además de su alto costo.

El único método directo utilizado en los estudios fue la concentración de las drogas en la orina y en la sangre. Esto tiene como ventaja facilitar una lectura fidedigna de la presencia del antimalárico en el organismo de la persona, por ello es un método invasivo, caro y que lleva tiempo para ser aplicado. Además, está la necesidad de control riguroso de la metabolización y de la excreción del fármaco y esto no garantiza la adhesión debido a la diferencia de eliminación de cada droga antimalárica. ${ }^{(14,15)}$

En estudios de adhesión al tratamiento, está indicado que los investigadores determinen la definición de adhesión que van a adoptar. El hecho de que en la mayoría de los estudios analizados los autores colocaran una definición operacional de la adhesión al tratamiento, permite que los resultados puedan ser comparados y que reflejen la adhesión/no adhesión que se pretende estudiar. Como la adhesión al tratamiento es un comportamiento del ser humano, cargado de subjetividad, exige que los investigadores estén atentos, pues corren el riesgo de sufrir sesgos en los resultados, caso de que los procedimientos metodológicos no sean cuidadosamente establecidos y seguidos.

La variabilidad en los resultados de prevalencia de la adhesión al tratamiento de la malaria encontrada en los estudios revisados puede explicarse por el hecho de que las investigaciones han sido realizadas en regiones y poblaciones diferentes y con esquemas diversos. Estudios que utilizaron el esquema cloroquina + primaquina, por ejemplo, presentaron tasas de prevalencia que variaron entre $62,2 \%$ y $76,2 \%$, mientras que las tasas de los que utilizaron el esquema artemeter + lumefantrina variaron entre $59,1 \%$ y $97,3 \%$.

El análisis de los factores contribuyentes a la adhesión y a la no adhesión al tratamiento de la malaria evidencia la necesidad de mayores inversiones en capacitación de los profesionales/agentes de salud y mejora en el acceso a los servicios. Por los resultados se acredita que educación en salud, capacitación de los profesionales de salud y garantía de acceso a medicamentos y servicios de salud promueven la adhesión al tratamiento de la malaria.

\section{CONCLUSIÓN}

Esta revisión identificó una gran cantidad de publicaciones sobre malaria, mas solamente fueron incluídos 27 estudios. Esto se debe principalmente al hecho de que solo estos tuvieran como foco principal la adhesión al tratamiento de los antimaláricos.

Los resultados mostraron que la mayoría de los estudios fue publicada en periódicos del área médica y realizada en África y en América Latina. El tipo de malaria más estudiado fue el Plasmodium falciparum y los esquemas terapéuticos cloroquina + 
primaquina; artemeter + lumefantrina y combinaciones de la sulfadoxina-pirimetamina con otras drogas. El principal objetivo de las investigaciones fue verificar la adhesión al tratamiento. Pocos estudios buscaron conocer los factores que contribuyen a la adhesión/no adhesión al tratamiento de drogas antimaláricas. Los niños fueron los sujetos más estudiados. Los autores utilizaron más de un método para medir la adhesión y el auto-relato estuvo presente en casi todos los estudios. La definición de adhesión estuvo relacionada con el seguimiento completo del tratamiento confirmado por el recuento de medicamentos.

Los resultados revelaron mucha variabilidad en las tasas de prevalencia de adhesión / no adhesión. Entre los factores contribuyentes a la adhesión al tratamiento destacaron mayor conocimiento de la enfermedad, gravedad de los síntomas, dosis supervisionada, gratuidad de los fármacos, uso de figuras pictóricas en el embalaje de los antimaláricos, y buenas orientaciones de los profesionales de salud en relación a los medicamentos y sus efectos. En relación a la no adhesión al tratamiento, destacaron los factores olvido, mejora de los síntomas, dificultad de acceso, efectos colaterales de los antimaláricos, falta de orientaciones y/u orientaciones incorrectas sobre los fármacos realizadas por el profesional de salud.

El estudio sistematizado de las investigaciones permitió conocer el estado actual de las publicaciones realizadas sobre la adhesión al tratamiento de la malaria y constatar que existen lagunas a ser rellenadas con la realización de futuras investigaciones. Sugerimos que se realicen estudios en el sentido de verificar la prevalencia de adhesión al tratamiento de la malaria en los países latino-americanos, considerando que son pocos los hasta ahora realizados. Investigaciones con ese propósito contribuirán al conocimiento de la magnitud del problema de la no adhesión al tratamiento de la malaria en esos países, principalmente si los autores se preocuparan en desarrollarlas con diseños metodológicos semejantes.

De la misma forma, son necesarias investigaciones que no solo identifiquen los factores contribuyentes de la adhesión/no adhesión cuantitativamente, sino que amplíen el conocimiento de estos factores por medio de abordajes cualitativos. Estudios desarrollados por medio de perspectiva cuantitativa son fundamentales y contribuyen mucho al aumento del conocimiento sobre la adhesión a los tratamientos. Sin embargo, el enfoque cualitativo puede complementar estos estudios en la medida en que posibilitan la comprensión del fenómeno de la adhesión en la perspectiva de los sujetos, valorizando sus experiencias y significados.

\section{REFERENCIAS}

1. Ministério da Saúde. Secretaria de Vigilância em Saúde. Ações de Controle da Malária: manual para profissionais de saúde na Atenção Básica. Brasília, Ministério da Saúde; 2005. p51.

2. World Health Organization. World Malaria Report 2010. Geneva: World Health Organization, 2011. p238.

3. Ministério da Saúde. Secretária de Vigilância Epidemiológica em Saúde. Departamento de Vigilância Epidemiológica. Guia prático de tratamento da malária no Brasil. Brasília, Ministério da Saúde. 2010. p36.

4. World Health Organization. Global Report on Antimalarial drug efficacy and drug resistence: 2000-2010. Geneva: World Health Organization. 2010. p115.

5. World Health Organization. Adherence to long-term therapies. Geneva: World Health Organization. 2003. p194. 
6. Fogg $\mathrm{C}$ et al. Adherence to a six-dose regimen of artemether-lumefantrine for treatment of uncomplicated Plasmodium falciparum malaria in Uganda. The American Journal of Tropical Medicine Hygiene. 2004; 71: 525-530.

7. Osterberg L, Blasche T. Adherence to medication. The New England Journal Medicine. 2005; 353-355.

8. World Health Organization. Guideline for treatment of Malaria. Geneva: World Health Organization. 2006; 2: p194.

9. Landry P, lorillo D, Darioli R, Burnier M, Genton B. Do travelers really take their mefloquine malaria chemoprophylaxis? Estimation of adherence by an electronic pillbox. Journal of Travel Medicine. 2006; 13: 8-14.

10. Reiners AAO, Azevedo RCS, Ricci HA, Souza TG. Adesão e reações de usuários ao tratamento da malária: implicações para a educação em saúde. Texto Contexto Enfermagem. 2010; 19: 81-89.

11. Farmer KC. Methods for Measuring and Monitoring Medication Regimen Adherence in Clinical Trials and Clinical. Practice Clinical Therapeutics. 1999; 21: 1074-90.

12. Nemes MIB et al. Avaliação da aderência ao tratamento por anti-retrovirais em usuários deambulatórios do sistema. Público de assistência à AIDS no Estado de São Paulo. Coordenação Nacional DST/AIDS, Ministério da Saúde. 2000.

13. Kuhlmann J; Wingender W; Kuppers J. Development of methods for compliance measurements. Bratislava Medical Journal-Bratislavske Lekarske Listy. 1998; 99: 2328.

14. O'Brien MK; Petrie K; Raeburn J. Adherence to medication regimens: Updating a complex medical issue. Medical Care Review. 1992; 49(4): 435-454.

\section{REFERENCIAS DE LA BIBLIOGRAFíA INVESTIGADA}

I. Yépez MC, Zambrano D, Carrasco F, Yépez R Factores asociados com El incumplimiento del tratamiento antipalúdico en pacientes ecuatorianos. Revista Cubana de Medicina Tropical. 2000; 52: 81-89.

II. Reiners AAO, Azevedo RCS, Ricci HA, Souza TG. Adesão e reações de usuários ao tratamento da malária: implicações para a educação em saúde. Texto \& Contexto Enfermagem. 2010; 19: 536-44.

III. Twagirumukiza M, Kayumba PC, Kips JG, Vrijens B, Stichele RV, Vervaet C, Remon JP, Van Bortel ML. Evaluation of medication adherence methods in the treatment of malaria in Rwandan infants. Malaria Journal. 2010; 9: 206-212.

IV. Simba DO, Kakoko DC, Warsame M, Premji Z, Gomes MF, Tomson G, Johansson E. Understanding caretaker's dilemma in deciding whether or not to adhere with referral advice after pre-referral treatment with rectal artesunate. Malaria Journal. 2010; 9:123-131.

V. Gerstl S, Dunkley S, Mukhtar A, Baker S, Maikere J. Successful introduction of artesunate combination therapy is not enough to fight malaria: results from an adherence study in Sierra Leone. Transactions of the Royal Society of Tropical Medicine and Hygiene. 2010; 104: 328-335.

VI. Kabanywanyi AM, Lengeler C, Kasim P, King'eng'ena S, Schlienger R, Mulure $\mathrm{N}$, Genton B. Adherence to and acceptability of artemether-lumefantrine as firstline anti-malarial treatment evidence from a rural community in Tanzania. Malaria Journal. 2010; I 9: 48-54.

VII. Congpuong K, Bualombai P, Banmairuroi V, Na-Bangchang K. Compliance with a three-day course of artesunate-mefloquine combination and baseline antimalarial treatment in an area of Thailand with highly multidrug resistance falciparum malaria. Malaria Journal. 2010; 9: 43-50. 
VIII. Souares A, Moulin P, Sarrassat S, Carlotti MP, Lalou R, Le Hesran JY. Selfreported data: a major tool to assess compliance with anti-malarial combination therapy among children in Senegal. Malaria Journal. 2009; 8: 257-263.

IX. Bell DJ, Wootton D, Mukaka M, Montgomery J, Kayange N, Chimpeni P, Hughes DA, Molyneux ME, Ward SA, Winstanley PA, Lalloo DG. Measurement of adherence, drug concentrations and the effectiveness of artemetherlumefantrine, chlorproguanil-dapsone or sulphadoxine-pyrumethamine in the treatment of uncomplicated malaria in Malawi. Malaria Journal. 2009; 8: 204214.

X. Beer N, Ali AS, Rotllant G, Abass AK, Omari RS, Al-mafazy AW, Björkman A, Källander K. Adherence to artesunate-amodiaquine combination therapy for uncomplicated malaria in children in Zanzibar, Tanzania. Tropical Medicine and International Health. 2009; 14: 766-774.

XI. Simba DO, Warsame M, Kimbute O, Kakoko D, Petzold M, Tomson G, Premji $Z$, Gomes M. Factors influencing adherence to referral advice following prereferral treatment with artesunate suppositories in children in rural Tanzania. Tropical Medicine and International Health. 2009; 14: 775-783.

XII. Souares A, Lalou R, Sene I, Sow D, Le Hesran JY. Factors related to compliance to anti-malarial drug combination: example of amodiaquine/sulphadoxine-pyrimethamine among children in rural Senegal. Malaria Journal. 2009; 8:118-125.

XIII. Khantikul N, Butraporn P, Kim HS, Leemingsawat S, Tempongko SBMA, Suwonkerd W. Adherence to antimalarial drug therapy among vivax malaria patients in northern Thailand. Journal of Health Population and Nutrition. 2009; 27: 4-13.

XIV. Souares A, Lalou R, Sene I, Sow D, Le Hesran JY. Adherence and effectiveness of drug combination in curative treatment among children suffering uncomplicated malaria in rural Senegal. Transactions of the Royal Society of Tropical Medicine and Hygiene. 2008; 102: 751-758.

XV. Depoortere E, Guthmann JP, Sipilanyambe N, Nkandu E, Fermon F, Balkan S, Legros $\mathrm{D}$. Adherence to the combination of sulphadoxine-pyrimethamine and artesunate in the Maheba refugee settlement, Zambia. Tropical Medicine and International Health. 2004; 9: 62-67.

XVI. Depoortere E, Salvador ETC, Stivanello E, Bisoffi Z, Guthmann JP. Adherence to a combination of artemether and lumefantrine (Coartem®) in Kajo Keji, southern Sudan. Annals of Tropical Medicine $\varepsilon$ Parasitology. 2004; 98: 635-637.

XVII. Grietens KP, Soto V, Erhart A, Ribera JM, Toomer E, Tenorio A, Montalvo TG, Rodriguez H, Cuentas AL, D'Alessandro U, Gamboa D. Adherence to 7-day primaquine treatment for the radical cure of $P$. vivax in the Peruvian Amazon. The American Journal of Tropical Medicine Hygiene. 2010; 82: 1017-1023.

XVIII. Conteh L, Stevens W, Wiseman V. The role of comunication between clients and health care providers: implications for adherence to malaria treatment in rural Gambia. Tropical Medicine and International Health. 2007; 12: 382-391.

XIX. Kachur SP, Khatib RA, Kaizer E, Fox SS, Abdulla SM, Bloland PB. Adherence to antimalarial combination therapy with sulfadoxine-pyrimethamine and artesunate in rural Tanzania. The American Journal of Tropical Medicine Hygiene. 2004; 71: 715-722.

XX. Fogg C, Bajunirwe F, Piola P, Biraro S, Checchi F, Kiguli J, Namiiro P, Musabe J, Kyomugisha A, Guthmann J. The American Journal of Tropical Medicine Hygiene. 2004; 71: 525-530. 
XXI. Reilley B, Abeyasinghe R, Pakianathar MV. Barriers to prompt and effective treatment of malaria in northern Sri Lanka. Tropical Medicine and International Health. 2002; 7: 744-749.

XXII. Duarte EC, Gyorkos TW. Self-reported compliance with last malaria treatment and occurence of malaria during follow-up in a Brazilian Amazon population. Tropical Medicine and International Health. 2003; 8: 518-524.

XXIII. Llanos-Zavalaga F, Cotrina AR, Campana OS. Factores asociados a La adherencia al tratamiento de malaria in Piura y Tumbes - Perú. Revista Peruana de Medicina Experimental y Salud Pública. 2001; 18: 63-70.

XXIV. Kolaczinski JH, Ojok N, Opwonya J, Meek S, Collins A. Adherence of community caretakers of children to pre-packaged antimalarial medicines (HOMAPAK®) among internally displaced people in Gulu district, Uganda. Malaria Journal. 2006; 5: 40-48.

XXV. Kimberly EM, Mwandama D, Jafali J, Luka M, Filler SJ, Sande J, Ali D, Kachur SP, Mathanga DP, Skarbinski J. Adherence to treatment with artemetherlumefantrine for uncomplicated malaria in rural Malawi. Clinical Infectious Diseases. 2011; 53: 772-779.

XXVI. Rahman MM, Dondorp AM, Day NPJ, Lindegardh N, Imwong M, Faiz MA, Bangali MA, Kamal MATM, Karim J, Kaewkungwal J, Singhasivanon P. Adherence and efficacy of supervised versus non-supervised treatment with artemether/lumefantrine for the treatment of uncomplicated Plasmodium falciparum malaria in Bangladesh: a randomized controlled trial. Transactions of the Royal Society of Tropical Medicine and Hygiene. 2008; 102: 861-867.

XXVII. Pereira, E.A, Ishikawa EAY, Fontes, CJF. Adherence to Plasmodium vivax malaria treatment in the Brazilian Amazon region. Malaria Journal. 2011; 10: 355. 